\title{
Análise da Estrutura e Conteúdo de uma Base de Medidas Visando ao Controle Estatístico de Processos de Software
}

\author{
Monalessa Perini Barcellos ${ }^{1,2}$, Gleison Santos ${ }^{3}$, Ana Regina Rocha ${ }^{1}$ \\ ${ }^{1}$ COPPE/UFRJ - Universidade Federal do Rio de Janeiro \\ Programa de Engenharia de Sistemas e Computação \\ Caixa Postal: 68511 - CEP 21945-970 - Rio de Janeiro, RJ, Brasil \\ ${ }^{2}$ UFES - Universidade Federal do Espírito Santo - Centro Tecnológico \\ Departamento de Informática - CEP 29075-910 - Vitória, ES, Brasil \\ ${ }^{3}$ UNIRIO - Universidade Federal do Estado do Rio de Janeiro \\ Departamento de Informática Aplicada - CEP 22290-240 - Rio de Janeiro, RJ, Brasil \\ monalessa@inf.ufes.br; gleison.santos@uniriotec.br; darocha@cos.ufrj.br \\ Resumo. $O$ alcance da alta maturidade, caracterizada nos níveis A e B do MR- \\ MPS e nos níveis 4 e 5 do CMMI, requer a implementação do controle estatís- \\ tico de processos, que somente pode ser realizado se forem utilizados medidas \\ e dados adequados a esse contexto. No entanto, muitas organizações que inici- \\ am as práticas necessárias à alta maturidade precisam adiar a implementação \\ do controle estatístico de processos devido à inadequação de suas bases de \\ medidas. Este artigo apresenta alguns resultados da análise realizada em uma \\ base de medidas de uma organização de software, visando à futura implemen- \\ tação do controle estatístico de processos.
}

\begin{abstract}
Achieving the high maturity, characterized at MR-MPS levels A and $B$ and at CMMI levels 4 and 5, includes the statistical process control implementation, which only can be carried out if there are measures and data suitable to this context. However, many organizations which start the high maturity practices have to retard the statistical process control implementation because their measures repositories are unsuitable. This paper presents some results obtained from the analysis of the measures repository of a software organization, aiming the statistical process control future implementation.
\end{abstract}

\section{Introdução}

Apesar do crescente número de estudos publicados relacionados à aplicação do controle estatístico em processos de software, há poucos registros que forneçam orientações práticas satisfatórias para que as organizações se preparem para sua implementação, visto que uma parte considerável dos estudos registrados foca em evidenciar a possibilidade e vantagens da aplicação do controle estatístico a processos de software ou propor abordagens de melhoria de processos de software baseadas nos princípios do controle estatístico. Assim, dada a ausência de um conjunto formal, consolidado e detalhado de diretrizes para a preparação e implementação do controle estatístico de processos de software, as organizações têm encontrado dificuldades, destacando-se a não adequação de suas bases de medidas à aplicação das técnicas estatísticas [Kitchenham et al., 2006; Weller e Card, 2008]. Nesse contexto, alguns autores [Sargut e Demirors, 2006; Boffoli et al., 2008; Curtis et al., 2008] afirmam que há mais trabalho para preparar a estrutura 
necessária para realizar o controle estatístico de processos do que para realizar o controle estatístico propriamente dito, pois a alimentação das bases de medidas com medidas que não são úteis ao controle estatístico retarda sua realização, uma vez que, primeiramente, deve ser realizada a adequação das medidas para, só então, aplicar as técnicas do controle estatístico de processos.

Considerando essa questão e visando à antecipação da adequação das medidas e dos dados para a implementação futura do controle estatístico de processos, a base de medidas de uma organização de software do Rio de Janeiro foi analisada durante a realização das práticas requeridas nos níveis C do MR-MPS [Softex, 2009b] e 3 do CMMI [Chrissis et al., 2006]. Também foram analisados os dados coletados a fim de verificar se eles estavam adequadas ao controle estatítico e se, eventualmente, algum dos processos já se encontrava sob controle estatístico. Neste artigo, por questões de confidencialidade, a organização é referenciada como organização $X$.

Os principais resultados obtidos nessa avaliação estão descritos neste artigo, que está organizado em três seções, além desta introdução. Na seção 2 são apresentados os resultados da avaliação da estrutura da base de medidas e do Plano de Medição da organização; na seção 3 são apresentados os resultados da avaliação dos dados da base de medidas, que foram utilizados na análise do comportamento dos processos; e, na seção 4 são apresentadas as considerações finais do artigo.

\section{Avaliação da Estrutura da Base de Medidas e do Plano de Medição}

Em 2008, ao iniciar a execução das práticas requeridas no nível 3 do CMMI, a organização $\mathrm{X}$ possuía uma base de medidas alimentada por dados de projetos realizados no nível de maturidade anterior, porém, sendo essa base não adequada às novas necessidades e à futura implementação do controle estatístico, a organização definiu uma nova base de medidas e um novo Plano de Medição, que foram submetidos à avaliação. A avaliação foi realizada considerando o grau de atendimento a um conjunto de requisitos para utilização de uma base de medidas no controle estatístico de processos de software, definidos em um trabalho realizado no contexto de uma tese de doutorado [Barcellos, 2009]. Foram avaliados o Plano de Medição (registrado em uma planilha eletrônica), as definições das medidas (registradas em planilhas eletrônicas relacionadas ao Plano de Medição) e a estrutura da base de medidas (modelada em uma ferramenta CASE usando Diagramas de Classes UML e Diagramas Relacionais).

O Plano de Medição da organização foi elaborado relacionando-se objetivos de negócio, objetivos de medição, necessidades de informação e medidas. Estavam registrados quatro objetivos de negócio e 22 objetivos de medição associados aos objetivos de negócio, sendo que 20 dos objetivos de medição tratavam do monitoramento de processos. Durante a avaliação do Plano de Medição foram detectadas inadequações no registro de alguns objetivos de medição, necessidades de informação e medidas, bem como no relacionamento entre esses elementos. Assim, foram sugeridas ações para a revisão do Plano de Medição, a fim de excluir, incluir ou alterar objetivos de medição, necessidades de informação e medidas e adequar os relacionamentos entre esses elementos. Na Figura 1, como exemplo, é apresentado um fragmento do Plano de Medição e observações realizadas durante sua avaliação.

Avaliado o Plano de Medição como um todo, foram avaliadas as medidas definidas, o que também revelou problemas. Essa etapa da avaliação teve início com a ava- 
liação do modelo de definição operacional utilizado pela organização, o qual incluía os seguintes campos: nome, descrição, mnemônico, valor base, limite superior, limite inferior, equação de cálculo, unidade de medida, procedimento de medição, procedimento de análise, responsável pela medição, responsável pela análise, entidade medida, periodicidade de medição e os flags ativa, obrigatória, atômica e automática. Foi, então, sugerida a inclusão das seguintes informações: propriedade da entidade medida, momento de medição, momento da análise, periodicidade da análise, tipo de escala e valores da escala. Após a avaliação do modelo da definição operacional, a avaliação individual das definições das medidas foi realizada e sua adequação foi determinada individualmente. Os principais problemas encontrados foram definições operacionais ambíguas, incompletas ou inconsistentes, ausência de identificação de medidas correlatas e alta granularidade das medidas definidas. Para resolver os problemas identificados, foram sugeridas ações para definir novas medidas, redefinir algumas e corrigir a definição de outras.

\begin{tabular}{|l|l|}
\hline Objetivo de Negócio & Incrementar o nivel atual de satisfação dos usuários. \\
\hline Objetivo de Medição & Monitorar o processo de Planejamento do Projeto \\
\hline Questão & Qual a precisão das estimativas de cronograma (prazo) nos projetos de desenvolvimento? \\
\hline Medidas & PEP (Prazo Estimado do Projeto), PRP (Prazo Real do Projeto) \\
\hline $\begin{array}{l}\text { Considerações } \\
\text { registradas na avaliação }\end{array}$ & $\begin{array}{l}\text { a) As medidas de prazo por fase estão definidas, mas não estão associadas à questão definida. } \\
\text { b) No conjunto de medidas não há medidas que forneçam as taxas de precisão das estimativas de prazo. }\end{array}$ \\
\hline & $\begin{array}{l}\text { a) Associar as medidas de estimativas de prazo por fase e de prazo real por fase à questão "Qual a } \\
\text { precisão das estimativas de cronograma (prazo) nos projetos de desenvolvimento?" e inclui-las no } \\
\text { conjunto de medidas relacionadas à questão definida. } \\
\text { b) Criar medidas para calcular a precisão das estimativas (taxas) do projeto, das fases e de atividades } \\
\text { (ou pelo menos macroatividades). }\end{array}$ \\
\hline
\end{tabular}

Figura 1. Fragmento do Plano de Medição e observações registradas na avaliação.

Por fim, foi avaliada a estrutura da base de medidas, composta por 25 tabelas. A avaliação revelou problemas considerados críticos para a realização do controle estatístico de processos, destacando-se a não identificação das versões dos processos executados nos projetos, a fraca caracterização dos projetos (classificados por um tipo e paradigma apenas) e a impossibilidade de armazenamento de informações de contexto para os dados coletados. Foram, então, sugeridas ações para que essas inadequações fossem corrigidas. Na Figura 2 é apresentado um fragmento do checklist de avaliação utilizado durante a avaliação da estrutura da base de medidas e algumas observações registradas.

\begin{tabular}{|c|c|c|c|c|}
\hline Requisitos & \multicolumn{3}{|c|}{ Avaliação* } & Considerações realizadas na avaliação \\
\hline $\begin{array}{l}\text { 1. A base de medidas apresenta-se } \\
\text { bem estruturada e seus dados são } \\
\text { integrados. }\end{array}$ & () A & (x) AP & ( ) NA & $\begin{array}{l}\text { A base de dados é única, mas é preciso rever sua estrutura, pois nem todas as } \\
\text { informações necessárias podem ser obtidas. É possivel que haja outros sistemas } \\
\text { de informação na organização que armazenam as informações necessárias. Nesse } \\
\text { caso, uma reestruturação e integração devem ser realizadas. }\end{array}$ \\
\hline $\begin{array}{l}\text { 1.1 A estrutura definida para a base } \\
\text { de medidas permite relacionar as } \\
\text { medidas definidas aos processos e } \\
\text { atividades da organização nos quais } \\
\text { a medição deve ser realizada. }\end{array}$ & () A & (x) AP & ( ) NA & $\begin{array}{l}\text { Os processos não estão armazenados explicitamente na estrutura da base de } \\
\text { medidas, porém, aparentemente, é possivel identificar as atividades que } \\
\text { compõem cada processo e adequar a base de medidas para registrar } \\
\text { explicitamente os processos, relacionando-os com as atividades que os } \\
\text { compõem. }\end{array}$ \\
\hline $\begin{array}{l}1.2 \text { A base de medidas é única ou } \\
\text { composta por diversas fontes } \\
\text { corretamente integradas. }\end{array}$ & $(x) A$ & () AP & ( ) NA & $\begin{array}{l}\text { A avaliação considerou que a base de medidas da organização é composta apenas } \\
\text { pela base cuja estrutura foi disponibilizada para avaliação, uma vez que nenhuma } \\
\text { outra foi mencionada. }\end{array}$ \\
\hline
\end{tabular}

- Legenda: $\mathrm{A}=$ Atende; $\mathrm{AP}=$ Atende Parcialmente; $\mathrm{NA}=\mathrm{Não}$ Atende

Figura 2. Fragmento da avaliação da estrutura da base de medidas.

\section{Análise do Comportamento dos Processos utilizando Dados da Base de Medidas}

Após submeter a estrutura da base de medidas e o Plano de Medição à avaliação e rece- 
ber os resultados, a organização realizou a revisão desses elementos e iniciou a alimentação da base de medidas com dados de projetos. Em 2009, após a organização obter sucesso na avaliação do nível C do MR-MPS e do nível 3 do CMMI, buscando um diagnóstico sobre o comportamento de alguns de seus processos com vistas aos níveis de maturidade superiores, os dados coletados para alguns processos foram submetidos às técnicas do controle estatístico de processos. Para isso, foram utilizados dados coletados de Outubro de 2008 a Agosto de 2009, de acordo com o Plano de Medição que, no momento da avaliação dos dados, contava com 63 medidas relacionadas aos 18 processos do Nível C do MR-MPS em sua versão 1.2 [Softex, 2009a].

O primeiro passo consistiu em selecionar os subprocessos cujos comportamentos, descrito pelos dados coletados para as medidas a eles associados, seriam analisados. A escolha dos subprocessos baseou-se em boas práticas de Engenharia de Software e na indicação presente no Guia de Implementação do MR-MPS [Softex, 2009a], citando o texto presente no CMMI [Chrissis et al., 2006], que indica que se deve selecionar pelo menos um subprocesso relevante por fase do ciclo de vida, um subprocesso relacionado à gerência de projetos e um relacionado aos processos de apoio. Assim, foram selecionados os subprocessos relacionados à detecção de defeitos, presentes em todas as fases do ciclo de vida e de grande importância para a organização, o subprocesso Planejamento do Projeto e o subprocesso de apoio Garantia de Qualidade do Processo e do Produto.

Identificados os subprocessos cujos comportamentos seriam analisados, o segundo passo foi identificar as medidas associadas a cada subprocesso cujos dados seriam avaliados à luz do controle estatístico. O primeiro conjunto de medidas analisado foi o relacionado a defeitos. Foram selecionadas, inicialmente, duas medidas para iniciar a análise: densidade de defeitos baixos encontrados na homologação externa e densidade de defeitos baixos encontrados nos testes de produto. Ao analisar os dados associados a essas medidas, percebeu-se que o volume de dados disponível era insuficiente para traçar os gráficos. O baixo volume dessas medidas foi devido à sua alta granularidade, uma vez que essas medidas eram coletadas uma única vez em cada projeto. Outras medidas foram selecionadas e apresentaram o mesmo problema. Para que elas pudessem ser utilizadas no controle estatístico, seria preciso que mais coletas tivessem sido realizadas. Como cada coleta dessas medidas representava um novo projeto, aconselhou-se à organização diminuir a granularidade das medidas e relacioná-las não a projetos, mas a itens menores (e, portanto, mais frequentes) como, por exemplo, casos de uso.

Em seguida, foram analisados os dados das medidas associadas ao subprocesso Planejamento de Projeto. Nesse caso, todas as taxas analisadas (por exemplo: precisão de prazo do projeto e precisão de custo do projeto) foram desconsideradas, pois estavam associadas aos projetos e foram coletadas uma única vez por projeto. Foram analisados, então, os indicadores IDC (Índice de Desempenho de Custos) e IDP (Índice de Desempenho de Prazo). O IDC é calculado dividindo-se o valor de VA (Valor Orçado do Trabalho Realizado) por CR (Custo Real do Trabalho Realizado). O IDP é calculado dividindo-se o valor de VA por VP (Custo Orçado do Trabalho Agendado/Planejado). A análise dos dados relacionados ao Planejamento de Projeto teve início traçando-se um gráfico de controle com todos os dados coletados para as medidas IDC e IDP em todos os projetos, havendo muitos valores disponíveis, pois eram realizadas pelo menos duas monitorações de cronograma por projeto em cada semana. Em uma primeira plotagem, onde os dados foram plotados individualmente, os pontos ficaram dentro dos limites estatísticos. Porém, puderam ser percebidas diversas variações no comportamento dos 
processos ao longo do tempo que indicam, na verdade, que o comportamento do processo pode estar variando com o tempo (apesar de os dados ainda estarem dentro dos limites estatísticos) e apresentando várias distribuições misturadas, como descrito por Florac e Carleton (1999). Foi, então, gerado um novo gráfico com os dados agrupados em subgrupos semanais, tendo sido identificados pontos onde foram percebidas mudanças no comportamento do processo (conforme testes de estabilidades sugeridos em [Florac e Carleton, 1999]), indicando, assim, a presença de baselines diferentes.

A Figura 3 apresenta o gráfico de controle obtido para o indicador IDP (o comportamento do indicador IDC é análogo e, devido a restrições de espaço, não é exibido). As linhas pontilhadas verticais da figura indicam os períodos onde houve mudança no comportamento dos processos, a saber: até Fevereiro/2009, final de Abril/2009, meio de Junho/2009, início de Agosto/2009. Para a análise do comportamento do processo, o primeiro período identificado no gráfico (até Fevereiro/2009) foi desconsiderado, pois informações demonstraram que ele representa, de fato, um período na organização no qual os processos e procedimentos de planejamento e monitoração do projeto ainda estavam sofrendo muitos ajustes devido à implantação dos processos de Nível C. Este também foi um período conturbado devido à iminência da avaliação, festas e recesso de final de ano, paralisações e atrasos nos projetos e férias de alguns colaboradores.

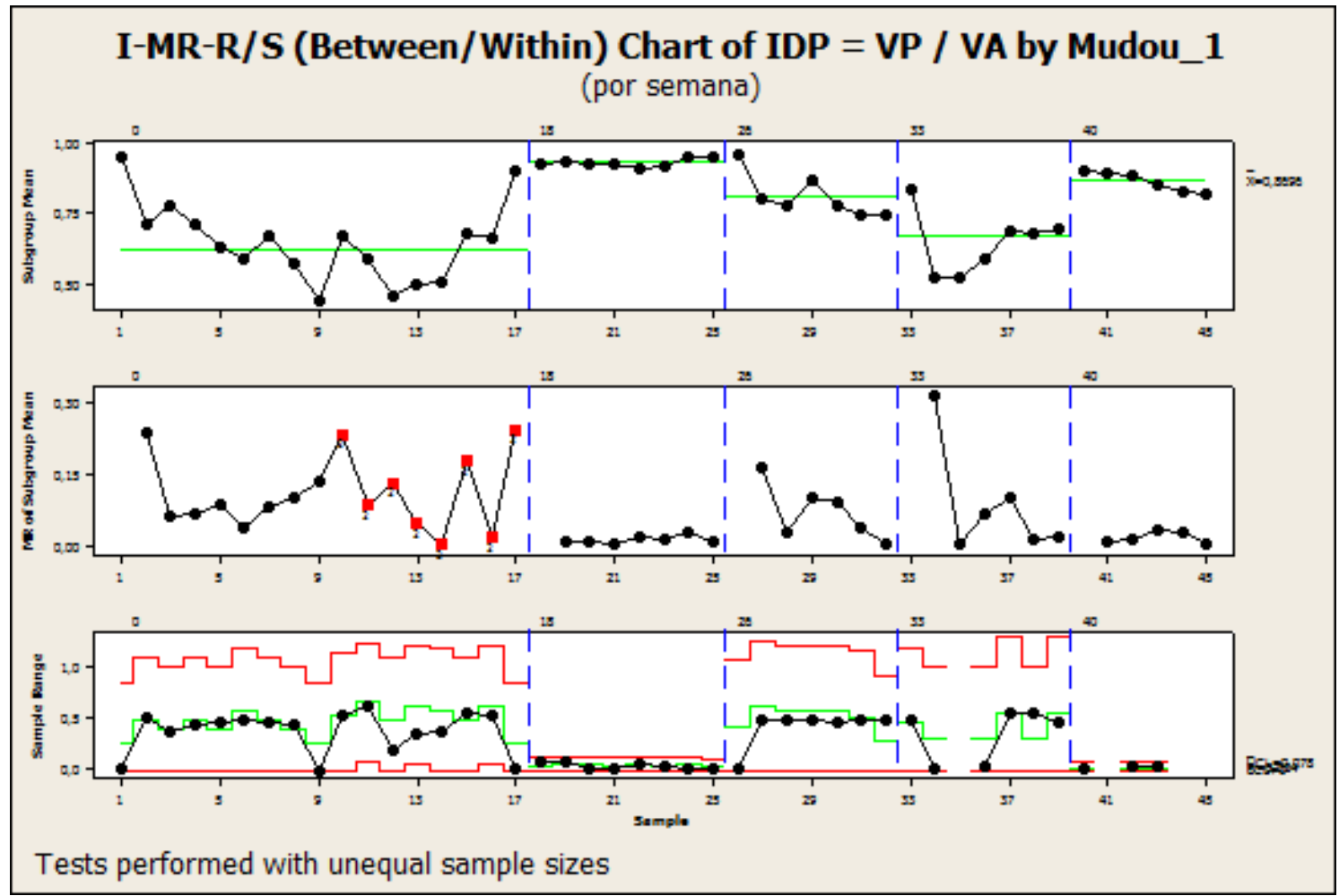

Figura 3. Dados da medida IDP aplicados em um gráfico de controle.

Nota: O primeiro gráfico representa os dados dos subgrupos (gráfico X-bar), o segundo, as médias móveis dos subgrupos (gráfico $\mathrm{mR}$ ) e o terceiro, a variação interna dos subgrupos (gráfico $S$ ).

Durante a análise, o Grupo de Processos salientou que só são considerados para os projetos custos referentes a Homens-Hora diretamente relacionados à quantidade de horas estimadas e as horas realizadas. Dessa forma, o indicador IDC não se presta tanto a analisar a efetividade da monitoração do projeto em si, mas, principalmente, ao processo de estimativa de esforço. Apesar da aparente estabilidade, é perceptível que o comportamento do subprocesso, descrito pelo indicador IDC, muda com muita frequên- 
cia. Dessa forma, sugeriu-se à empresa um olhar atento para a sua execução e controle.

Todas as medidas inicialmente selecionadas em relação à Garantia de Qualidade do Processo e do Produto tiveram que ser desconsideradas (taxa de avaliações de processo realizadas, taxa de realização de auditorias externas e taxa de avaliações de produto realizadas) devido à insuficiência de dados provocada pela alta granularidade das medidas. Foram, então, derivadas novas medidas para capturar a taxa de não conformidades encontradas nas avaliações de produto. Para isso, foram considerados o número de critérios avaliados em cada laudo de qualidade de produto (NCQP), o número de critérios registrados como fora de escopo na avaliação (NCFE) e o número de critérios para os quais o responsável pela avaliação identificou uma não-conformidade (NCNC). A partir dessas medidas foi calculada a medida taxa de não conformidades em avaliações do PPQA no Relatório de Monitoração dos Projetos, dada (em percentual) por NCNC / (NCQP - NCFE). Os dados obtidos para a medida foram, então, plotados em gráficos de controle XmR, como apresentado na Figura 4. As linhas pontilhadas verticais representam os pontos onde houve mudança no comportamento do processo, sendo: até Fevereiro/2009 e final de Abril/2009.

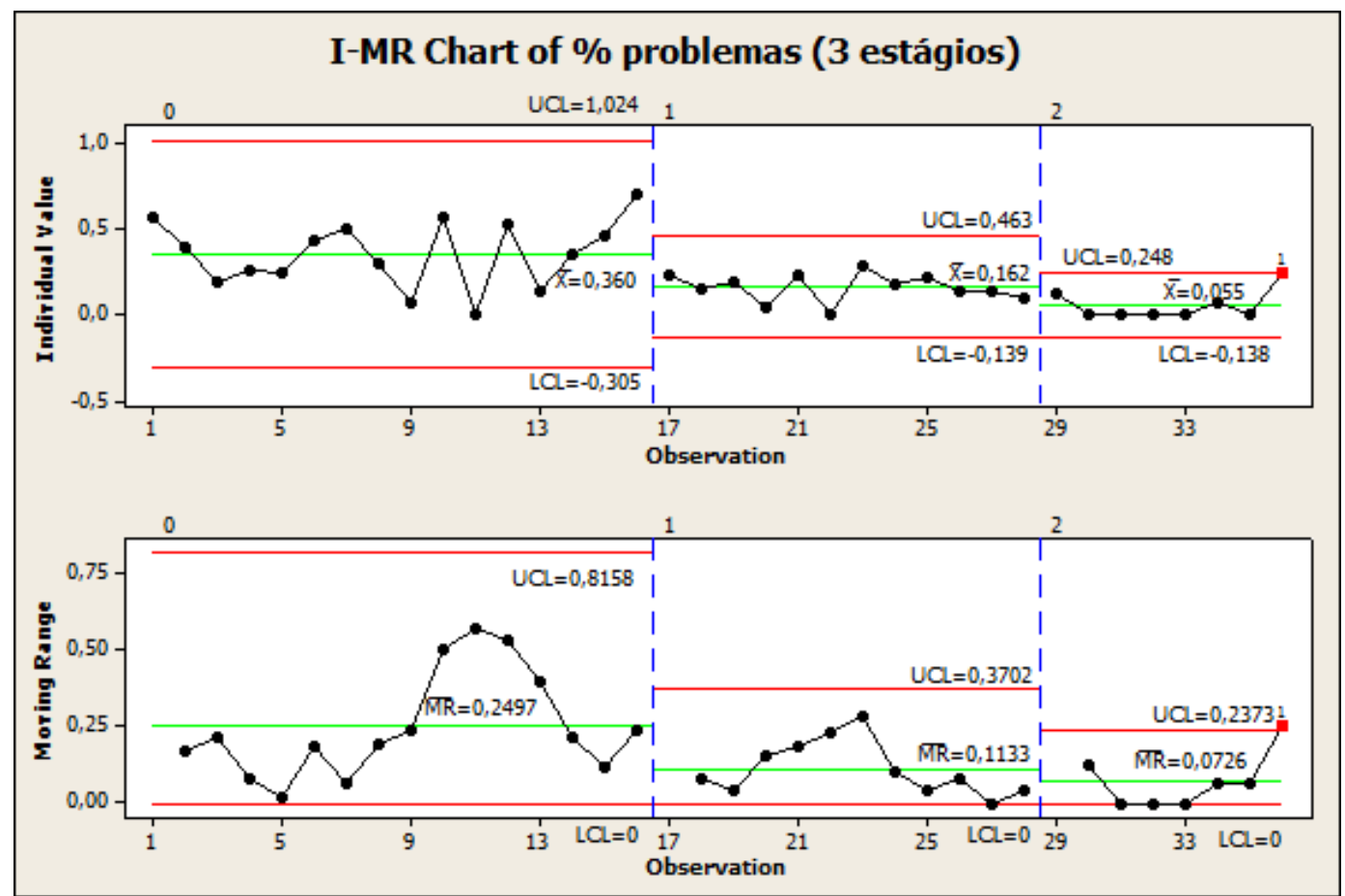

Figura 4 - Dados da medida taxa de não conformidades em avaliações do PPQA no Relatório de Monitoração dos Projetos plotados em um gráfico XmR.

Observando-se o gráfico de controle, percebe-se que o comportamento do processo de Garantia da Qualidade do Produto e do Processo, quando descrito pela medida taxa de não conformidades em avaliações do PPQA no Relatório de Monitoração dos Projetos, apresenta-se, aparentemente, sob controle nos três períodos considerados, exceto pelo último ponto (ocorrido em Junho/2009) do gráfico de médias móveis (gráfico inferior da Figura 4). Também é possível notar que a variação do comportamento foi gradativamente diminuindo (a média da primeira distribuição foi $0,36 \%$, depois de $0,162 \%$ e, por fim, $0,055 \%$ ). As avaliações que deram origem aos dados coletados no último período coincidem com o período em que a organização estava altamente comprometida com o resultado das avaliações finais do MR-MPS e do CMMI. Os procedi- 
mentos adotados para realizar as avaliações e os critérios dos laudos não foram alterados, mas os líderes de projeto se empenharam em elaborar os relatórios de monitoração com maior precisão para que fossem aprovados o mais rápido possível e, assim, não trouxessem impacto ao andamento dos projetos e à avaliação nos níveis dos modelos.

Voltando ao ponto de instabilidade anteriormente citado (o último ponto do gráfico de médias móveis), o gráfico mostra que houve uma avaliação com taxa 0 (zero) e $\operatorname{logo}$ em seguida outra com $0,25 \%$. Buscando entender essa variação, foi conduzida uma investigação para determinar se esse era um comportamento anormal isolado ou se representava uma nova mudança no comportamento do processo. Para isso, foram analisados novos dados coletados a partir de 25/06/2009, que revelaram que, realmente, houve outra mudança no comportamento do processo, tendo sido a média dos pontos elevada de 0,055 para 0,196 (356\%) e o limite superior de 0,248 para 0,673 (271\%).

Inicialmente, imaginou-se que essa mudança teria sido causada por relaxamento dos líderes de projeto após as avaliações CMMI e MR-MPS, que teria feito com que mais problemas passassem a ser gerados e capturados pela qualidade. Entretanto, descobriu-se outra informação de contexto relevante. Durante o novo período analisado, foram coletados dados de apenas um projeto não considerado nas análises anteriores, uma vez que teve início no final de Maio/2009 (ou seja, todos os relatórios de monitoração foram produzidos a partir de Junho/2009). Uma investigação junto à organização revelou que esse comportamento foi devido ao fato de que a equipe do projeto estava 'burlando' algumas atividades do processo (por exemplo, não respeitavam o momento das avaliações de qualidade e paralelizavam atividades impropriamente) sem o conhecimento da área de qualidade. Isso fez com que outras medidas também apresentassem problemas. Dessa forma, decidiu-se desconsiderar os dados coletados nesse projeto da análise do comportamento do subprocesso por serem caracterizados como outliers.

Analisando os dados coletados e os gráficos das Figuras 3 e 4 pode-se perceber que não há correlação entre as distribuições, no entanto, os subprocessos apresentam mudanças de comportamento em momentos equivalentes, o que pode significar que essas mudanças foram originadas nas mesmas causas.

\section{Considerações Finais}

Em organizações de software, o controle estatístico é utilizado para apoiar a análise do comportamento de processos. Para isso, são utilizadas técnicas estatísticas para analisar os dados coletados para medidas ao longo de projetos, a fim de conhecer seu comportamento, determinar seu desempenho e, então, fornecer diretrizes, caso necessário, para realizar ações corretivas e de melhoria que levem os processos a alcançarem os objetivos para eles estabelecidos [Chrissis et al., 2006]. As técnicas estatísticas e de resolução de problemas envolvidas no controle estatístico de processos requerem que as medidas definidas e os dados coletados sejam adequados à aplicação nesse contexto. No entanto, de acordo com os registros da literatura, esse não tem sido um fato comum, tendo sido relatados diversos problemas relacionados às bases de medidas que impossibilitam ou retardam a implantação do controle estatístico de processos [Barcellos, 2009].

Este artigo apresentou os principais resultados da análise realizada em uma base de medidas de uma organização de software que implementou as práticas do nível $\mathrm{C}$ do MR-MPS e do nível 3 do CMMI e desejava um diagnóstico para a implementação futura do controle estatístico de processos. Apesar de a avaliação da estrutura da base de medidas e do Plano de Medição ter identificado alguns problemas na definição das 
medidas (por exemplo, a alta granularidade das medidas definidas), durante a avaliação do comportamento dos processos utilizando os dados coletados para as medidas percebeu-se que o problema foi solucionado em alguns casos e persistiu em outros.

Atualmente a organização $\mathrm{X}$ está iniciando a implementação de processos visando a uma avaliação MR-MPS nível A. O processo de desenvolvimento foi revisto e está sendo componentizado. Uma nova versão do Plano de Medição da organização foi elaborado para garantir que as medidas definidas e os dados para elas coletados sejam adequados ao controle estatístico, atendendo, especialmente, à granularidade necessária (principal problema detectado anteriormente) e possuindo informações de contexto relevantes. Uma nova versão da base de medidas será construída ainda este ano.

É importante ressaltar que, apesar de algumas medidas indicarem que os subprocessos estão aparentemente sob controle estatístico, ainda é cedo para dizer que a organização está pronta para uma avaliação nos níveis mais altos de maturidade, uma vez que ainda há questões a serem abordadas e adequações a serem realizadas. Além disso, a análise do comportamento dos processos revelou que as mudanças ainda são frequentes. Por fim, vale salientar que a alta maturidade no desenvolvimento de software é obtida a partir de muito esforço associado à implementação de diversas práticas de Engenharia de Software, não se limitando à análise do comportamento dos processos através das técnicas do controle estatístico.

\section{Referências Bibliográficas}

BARCELlOS, M. P. (2009) "Uma Estratégia para Medição de Software e Avaliação de Bases de Medidas para Controle Estatístico de Processos em Organizações de Alta Maturidade", Tese de Doutorado, COPPE/UFRJ - Universidade Federal do Rio de Janeiro.

BOFFOLI, N., BRUNO, G., CAIVANO, D., MASTELlONI, G. (2008) "Statistical Process Control for Software: a Systematic Approach" In Proceedings of the 2008 ACM-IEEE International Symposium on Empirical Software Engineering and Measurement, Kaiserslautern - Germany, p. 327-329.

CHRISSIS, M. B., KONRAD, M., SHRUM, S. (2006) "CMMI (Second Edition): Guidelines for Process Integration and Product Improvement", Addison-Wesley.

CURTIS, B., REIFER, D., SESHAGIRI, G. V., HIRMANPOUR, I., KEENI, G. (2008) "The Case for Quantitative Process Management" IEEE Software, v. 25, n. 3, p. 24-28.

FLORAC, W. A., CARLETON, A. D. (1999)" Measuring the Software Process: Statistical Process Control for Software Process Improvement", Addison Wesley.

KITCHENHAM, B., KUTAY, C., JEFFERY, R., CONNAUGHTON, C. (2006) "Lessons Learnet from the Analysis of Large-scale Corporate Databases" In Proceedings of the 28th International Conference on Software Engineering - ICSE'06, Shanghai, China, p. 439-444.

SARGUT, K. U., DEMIRORS, O. (2006) "Utilization of Statistical Process Control (SPC) in Emergent Software Organizations: Pitfalls and Suggestions" Software Quality Journal, v. 14 , n. 5 , p. 135-157.

SOFTEX (2009a) "MPS.BR: Melhoria de Processo do Software Brasileiro - Guia de Implementação", Disponível em: http://www.softex.br/mpsbr

SOFTEX (2009b) "MPS.BR: Melhoria de Processo do Software Brasileiro - Guia Geral", Disponível em: http://www.softex.br/mpsbr.

WELLER, E. F., CARD, D. (2008) "Appling SPC to Software Development: Where and Why" IEEE Software, v. 25, n. 3, p. 48-50. 